\title{
Passive bistatic radar using digital terrestrial television broadcasting signal for subsurface target detection
}

\author{
Weike Feng ${ }^{1 *}$, Jean-Michel Friedt ${ }^{2}$, Suyun Wang ${ }^{1}$, and Motoyuki Sato ${ }^{3}$ \\ ${ }^{1}$ Graduate School of Environmental Studies, Tohoku University, 980-8579, Sendai, Japan \\ ${ }^{2}$ Time \& Frequency department, FEMTO-ST, 25000, Besancon, France \\ ${ }^{3}$ Center for Northeast Asian Studies, Tohoku University, 980-8576, Sendai, Japan \\ Email: feng.weike.q4@dc.tohoku.ac.jp
}

\begin{abstract}
A passive bistatic radar (PBR) system, which exploits the digital terrestrial television broadcasting (DTTB) emitter as the illumination source without using a dedicated transmitter, has been developed for subsurface target detection. Its main features are low system cost, high system flexibility, and no need for frequency allocation. In this paper, at first, the fundamental concept, system components, and basic signal processing methods are introduced. The characteristics of the adopted DTTB signal with the Japan ISDB$\mathrm{T}$ standard are evaluated. Then, one of the key techniques for ISDB-T DTTB signal based PBR, i.e., reference signal refinement (reconstruction and modification), is presented to suppress the strong sidelobes in the range-Doppler domain caused by the waveform properties and multipath echoes. Preliminary experimental results are shown to demonstrate the basic functions of the developed radar system. It has been found that the reference signal refinement method can work well in practice and a metallic plate buried in the sand can be effectively detected by the developed PBR system. At last, potential improvement directions of the PBR system for subsurface target detection, e.g., combing multiple bands, working with cooperative targets, and using super-resolution algorithms, are provided. Index Terms - Passive radar, digital terrestrial television broadcasting, subsurface target detection.
\end{abstract}

\section{Introduction}

Passive bistatic radar (PBR), which exploits the existing illuminator of opportunity (IO) instead of a dedicated radar transmitter, has attracted a lot of attention over the last decades because of its low cost, low vulnerability, high freedom, no need for frequency allocation, and reduced electromagnetic pollution to the environment [1-3]. Different types of ground and space IOs, including frequency modulated (FM) broadcast emitter, digital audio broadcasting (DAB) transmitter, cell phone base station (GSM/3G), global navigation satellite system (GNSS), digital terrestrial television broadcasting (DTTB) emitter, digital communication networks (such as WiFi), digital broadcasting satellite (DBS) for television transmission, and existing surveillance radar or synthetic aperture radar (SAR) systems, have been exploited in PBR to detect, track and image air or coastal targets. Various PBR techniques, such as direction of arrival (DOA) estimation, SAR imaging, inverse SAR (ISAR) imaging, coherent change detection, and displacement estimation have been developed.

For different PBR applications, frequency, bandwidth, and transmitting power may be the most basic properties of the employed IO signals that should be selected to satisfy the requirements. In this research, subsurface target detection, which is commonly realized by using ground penetrating radar (GPR) techniques [4-5], is considered to be carried out by using the PBR technique. Previously, with GNSS signal (1.5 GHz) and WiFi signal (2.4 GHz), subsurface targets have been successfully detected by PBR, as presented in [6] and [7], respectively. As we know, the rule of thumb for subsurface detection is that: the stronger signal with a lower frequency normally has a deeper penetration capability. With a lower frequency (around $500 \mathrm{MHz}$ ) and a higher power, DTTB signal, which also has a wider bandwidth than its counterparts (i.e., FM and DAB at the VHF bands), seems more suitable for subsurface detection applications than GNSS and WiFi signals. Therefore, a PBR system using the DTTB signal as the source has been developed and is presented in this paper to evaluate its practical subsurface target detection performance.

Existing commercial IO signals are originally used for communication, navigation, or broadcasting purpose, so their waveforms are not designed for radar application. To satisfy their own requirements, the waveforms of commercial IO signals always have some specific properties, such as filtered amplitudes, spectral gaps, preamble signals, pilot signals, and guard intervals. Therefore, for PBR applications, apart from the frequency, bandwidth, and transmitting power, the waveform of the adopted IO signal should also be assessed. To this 
end, the ambiguity function, which is defined as a two-dimensional (2D) function of the time delay and Doppler frequency and can be used to determine the range and Doppler resolutions of a specific waveform, is always used. Based on the ambiguity function, in this paper, the characteristics of the DTTB signal with the integrated services digital broadcasting terrestrial (ISDB-T) standard used for the developed PBR system have been analyzed.

Since the IO signal is not within control, two receiving channels, i.e., reference and surveillance channels, are normally needed for PBR. The reference channel is used to obtain a template of the transmitted IO signal by an antenna directly oriented to the IO (e.g., TV tower). The surveillance channel is used to sample the reflections from the targets. Then, the reference and surveillance signals are crosscorrelated to calculate the delays and Doppler frequencies of the targets. To achieve optimal matched-filtering performance, the reference signal should be as clean as possible. However, apart from the measurement noise, the reference channel will also receive the reflections from stationary and moving targets. Therefore, to get a pure reference signal to reduce the interferences of these reflections and improve the target detection performance, reference signal reconstruction should be carried out by the demodulation and remodulation process [8]. Furthermore, with simple modifications of the reconstructed reference signal, the range-Doppler sidelobes caused by the properties (as mentioned above) of the IO waveform can be suppressed [9]. Therefore, for the developed PBR system, a reference signal refinement method has been proposed and validated in this paper.

This paper is organized as follows. In Section II, the developed PBR system, the properties of the adopted DTTB signal, and the basic signal processing methods are presented. In Section III, a reference signal refinement method is presented and the corresponding processing methods of the surveillance signal are given. In Section IV, preliminary experiment results are shown to validate the developed system and the proposed methods. Some discussions of the performance improvement of the developed PBR system for subsurface target detection are provided. Finally, section V concludes this paper.

\section{Fundamentals of the developed PBR system}

As shown in Figure 1, the developed PBR system mainly consist of a reference antenna (Yagi-Uda) facing to the TV tower to receive the direct signal, a surveillance antenna (Bowtie) facing to the ground to receive the target reflections, a GNSS positioning system (Trimble NetR9) used to locate the system, a universal software radio peripheral platform (Ettus Research USRP B210) used to sample the data from two channels, and a PC used for data acquisition control, real-time result display, and post signal processing.
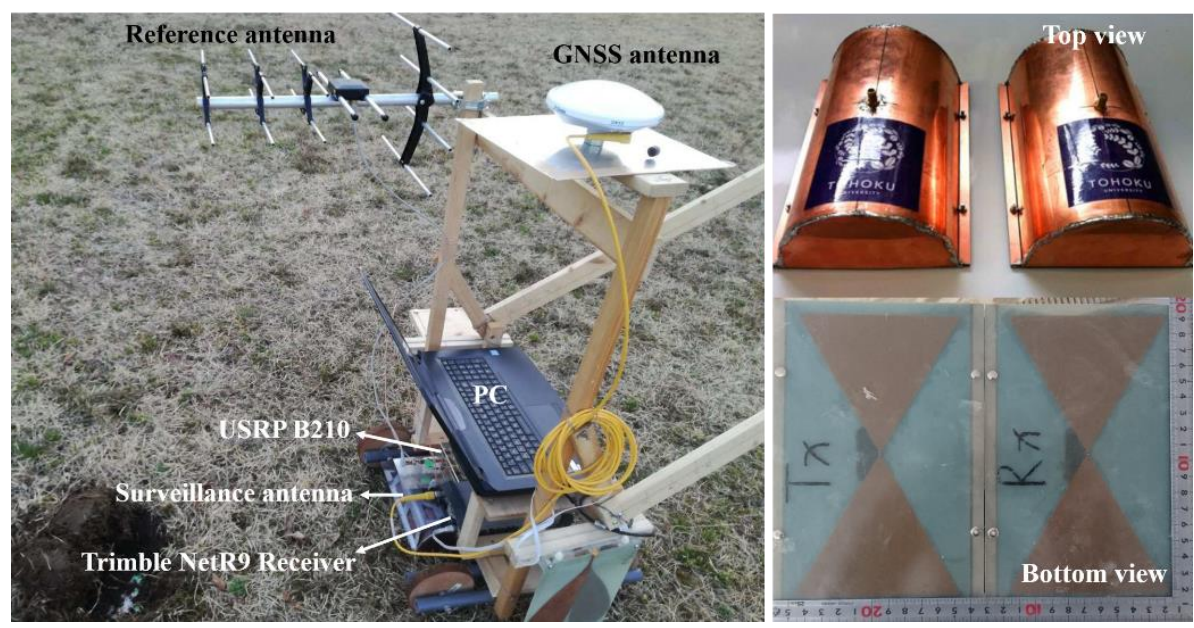

Figure 1. (Left) the developed PBR system for subsurface target detection (the Vivaldi antenna on the side is not used is this paper) and (right) Bowtie antennas (one of them is used as the surveillance antenna in this paper).

The Yagi-Uda antenna is low cost and readily obtained on the market. It is suitable to be used as the reference antenna for DTTB based PBR applications, as demonstrated by us previously for moving target range-Doppler map generation [10] and stationary target SAR imaging [11]. The Bowtie antenna with a dimension of $25 \mathrm{~cm} \times 17 \mathrm{~cm} \times 8 \mathrm{~cm}$ is initially used for GPR application [12]. It has a copper 
shield for minimization of the reception of the DTTB signal directly reach to the antenna. The application of USRP is driven not only by the flexibility and reconfigurability of the software compared to the hardware but also by the lower cost of consumer electronics. The flexibility of USRP allows for the use of single affordable hardware in a wide range of fields. In this paper, the open-source GNURadio [13] framework is used as the development toolkit to implement software radios and display the range compression results in real-time. The PBR system is precisely positioned by the Real-time kinematic (RTK) technique using RTKLIB [14] and the 2D Kalman filter algorithm. The positions of the PBR system can be used for the following azimuth compression to generate a focused 3D target image. As shown in Figure 2, six DTTB channels using the ISDB-T standard are transmitted by three TV towers in Sendai. In this paper, the Miyagi Television Broadcasting (MMT) channel with a central frequency of $539.143 \mathrm{MHz}$ is evaluate and used.
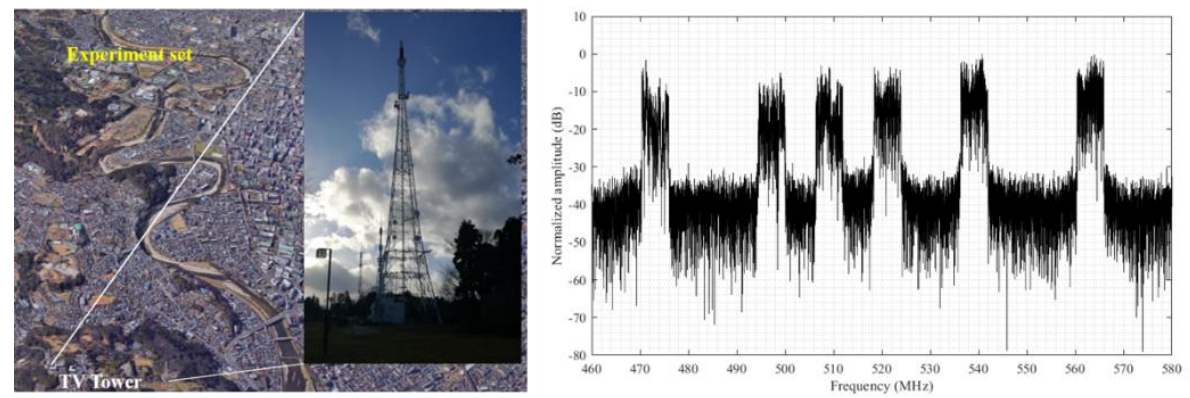

Figure 2. (Left) TV towers and (right) DTTB signal spectrum in the area of Sendai, Japan.

To evaluate its properties, the ambiguity diagram of a real-sample DTTB signal is calculated by (1) and shown in Figure 3.

$$
\chi\left(\tau, f_{d}\right)=\int_{-\infty}^{\infty} s(t) s^{*}(t-\tau) e^{-j 2 \pi f_{d} t} d t
$$

In (1), $s(t)$ is the DTTB signal, $\tau$ is the time delay, $f_{d}$ is the Doppler frequency, and $(\cdot)^{*}$ denotes complex conjugate. For zero Doppler frequency, i.e., $f_{d}=0$, the ambiguity function degrades to the auto-correlation function of $s(t)$. It can be seen from Figure 3 that the used DTTB signal has a thumbtack-like ambiguity diagram, indicating its suitability for PBR applications. However, it can also be seen that there are many undesired sidelobes in the delay-Doppler domain, which are caused by the pilot signal and guard interval of the DTTB signal. The scattered pilot carriers are uniformly distributed in the frequency domain with a constant spacing of $11.905 \mathrm{kHz}$, resulting in sidelobes in the time domain every $84 \mu \mathrm{s}$. The pilot signal is regularly repeated in the time domain with an interval of $4536 \mu \mathrm{s}$, causing sidelobes in Doppler every $220.46 \mathrm{~Hz}$. The guard interval, which is the repeat of the end of an OFDM symbol, generates sidelobes every $1008 \mu$ s. These sidelobes may cause negative influences on target detection. Therefore, some suppression methods should be adopted. However, for short-range (e.g., subsurface target detection considered in this paper) and slow target applications, these sidelobes can be ignored. In this paper, the introduction of these sidelobes is mainly used to demonstrate the performance of reference signal refinement.

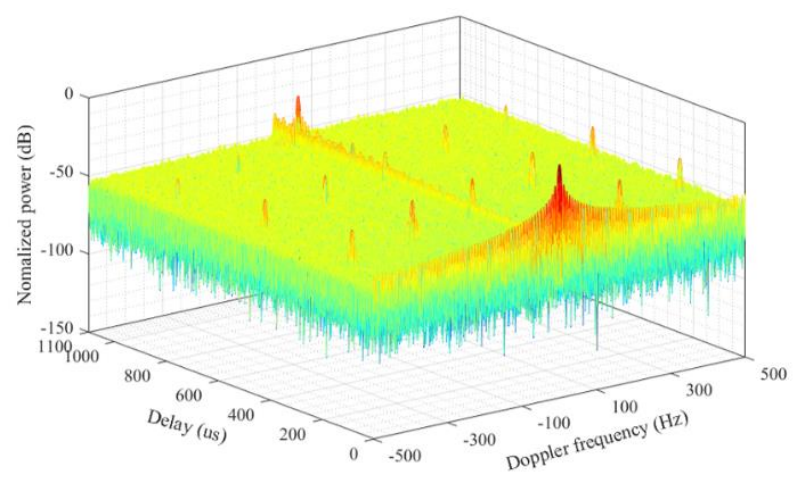

Figure 3. Ambiguity diagram of the real-sampled DTTB signal.

For subsurface target detection, the developed PBR system is moved on the ground to generate a 2D synthetic aperture. GNSS data, 
reference signal, and surveillance signal are simultaneously sampled. Considering the speed of movement is low (the Doppler shift of the received signal can thus be ignored), the coherent integration time (CPI) can be randomly selected for range compression given the limitation imposed by the azimuth aliasing (i.e., the antenna distance between two adjacent CPIs should be smaller than half of the wavelength). For the $l$-th CPI, after obtaining the refined reference signal $s_{\text {ref }}(t)$ (which will be detailed in Section III), range compression can be conducted by calculating the cross-correlation function between $s_{r e f}(t)$ and the surveillance signal $s_{\text {surv }}(t)$, as

$$
\chi^{l}(\tau)=\int_{0}^{T_{\text {int }}} s_{\text {surv }}^{l}(t)\left(s_{\text {ref }}^{l}\right)^{*}(t-\tau) d t
$$

where $l=1,2, \ldots, L$ and $T_{\text {int }}$ denotes CPI. Then, azimuth compression can be conducted by using the back projection algorithm as

$$
\sigma(x, y, z)=\sum_{l=1}^{L} \chi^{l}\left[\tau_{l}(x, y, z)\right]
$$

where $\tau_{l}(x, y, z)$ is the $l$-th delay of the target at $(x, y, z)$. It should be noted that, in the current study step, as the range resolution is too low to generate a 3D subsurface target image, only range compression is performed based on (2) and the maximal amplitude is picked from each CPI to detect the target.

\section{Reference signal refinement}

In this Section, reference signal refinement of the ISDB-T DTTB signal is discussed. To this end, the basic structure of the ISDB-T signal should be understood. In the following, the modulation scheme, guard interval, and non-data carriers, which are important for reference signal reconstruction, are introduced.

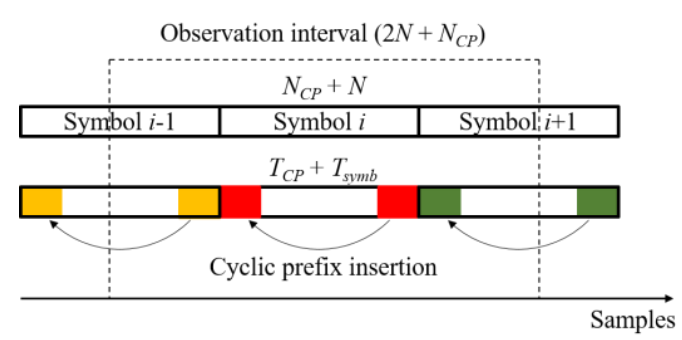

Figure 4. Structure of the OFDM signal with cyclic prefix insertion.

OFDM modulation scheme is used by ISDB-T. The information is carried by $N$ equally spaced sinusoids $(N=8192$ for the mode- 3 ISDB-T standard in Japan), all these sinusoids (sub-carriers) will be transmitted simultaneously, which is conducted by the inverse Fourier transform with a sampling rate of $f_{s}=512 / 63 \approx 8.126 \mathrm{MHz}$ of a set of constellation symbols. The duration of one OFDM symbol is $T_{\text {smyb }}=N / f_{s}=1008 \mu \mathrm{s}$ and the carrier spacing is $\Delta f=992.06 \mathrm{~Hz}$. After modulation, a cyclic prefix (CP), which is a copy of the last part of the OFDM symbol, is prepended to the beginning, as shown in Figure 4. The length of the CP can be 1/4, 1/8, 1/16, or 1/32 of the symbol length. In Japan, the CP duration is $T_{C P}=1008 / 8=126 \mu \mathrm{s}$, which is known as the guard interval (GI). The function of GI is to combat the inter-symbol interferences caused by the propagation channel. As shown previously, GI causes sidelobes every $T_{s m y b}=1008$ $\mu$ s. Not all 8192 carriers, but only $N_{U}=5617$ (useful) active carriers, are used for the information transmission. Therefore, the useful bandwidth of the ISDB-T signal is $B=N_{U} \Delta f \approx 5.57 \mathrm{MHz}$. The useful spectrum is then sub-divided into 13 segments, each of which has 432 carriers, while the left carrier is acting as the continual pilot (CP). The main difference of ISDB-T from the European DVB-T standard is the band segmented transmission OFDM (BST-OFDM) approach, based on which different segments (different layers) can transmit different transport streams (TSs) and use different modulation schemes. For 5617 active carriers, $N_{D}=384 \times 13=4992$ carriers are used for data transmission, while other carriers are used to transmit modulation parameters to assist the demodulation and decoding process in the receiver. $N_{T M C C}=4 \times 13=52$ transmission multiplexing configuration control (TMCC) carriers, which are always at the fixed positions, are used to transmit the modulation parameters of each segment. Since 204 bits need to be transmitted, 204 OFDM symbols, which form an OFDM frame, are required to receive all the modulation parameters. Besides, $N_{S P}=36 \times 13=468$ scattered pilots (SP) are used to help the receiver conducting channel equalization. The frequency spacing of SP is about $992.06 \times 12=11.905 \mathrm{kHz}$, 
resulting in sidelobes in the range direction every $84 \mu \mathrm{s}$, as shown in Figure 3. Different from the TMCC carrier, in order to avoid the pathological situations, the positions of SP carriers are changed from symbol to symbol with a period of 4 OFDM symbols. The periodical SP signal has an interval of $4 \times\left(T_{\text {smyb }}+T_{C P}\right)=4536 \mu$ s, causing sidelobes in the Doppler domain every $220.46 \mathrm{~Hz}$, as shown in Figure 3 . Moreover, $N_{A C}=8 \times 13=104$ auxiliary channel (AC) carriers are designed to convey additional information on the modulating signaltransmission control. At last, there is a single CP carrier at the highest frequency, which is different from the DVB-T standard that can use the CPs for frequency offset correction.

Based on above-introduced knowledge, the method used to refine the reference signal is described in the following, which mainly includes signal demodulation, remodulation, and modification. The presented method may not be the optimal one, but it is simple and works well for the data at hand. It should be pointed out that the used method is mainly based on the open and free ISDB-T receiver implemented in GNURadio [15]. However, specified to the PBR applications, some simplifications and modifications are performed.

\section{(1) OFDM acquisition}

The first step for ISDB-T signal demodulation is to know when an OFDM symbol starts, based on which $N=8192$ samples can be acquired. This step is called OFDM acquisition and, since the CP signal is the same as the last part of an OFDM symbol, it can be conducted by the maximum likelihood (ML) based method [16] with every $\left(2 N+N_{C P}\right)$ samples, as shown in Figure 4, given by

$$
\tilde{\theta}_{M L}=\underset{\theta}{\arg \max }\left\{\left|\sum_{n=\theta}^{\theta+N_{C P}-1} s(n) s^{*}(n+N)\right|-\frac{\rho}{2} \sum_{n=\theta}^{\theta+N_{C P}-1}|s(n)|^{2}+|s(n+N)|^{2}\right\}
$$

where $N_{C P}=N / 8=1024$ is the length of the CP, $s(n)$ is the $n$-th received signal sample, and $\rho=S N R /(1+S N R)$. When the required parameters used in (4) are known in advance, a triangle shape can be obtained and the peak indicates the initial sample of an OFDM symbol. On the contrary, when the basic parameters are unknown, a plateau pattern instead of a triangle pattern is obtained and the starting point of an OFDM symbol cannot be correctly estimated.

\section{(2) Frequency correction}

Once the boundaries of an OFDM symbol are estimated, i.e., time offset has been corrected, frequency offset caused by the difference between transmitter and receiver local oscillator frequencies should be corrected, which mainly includes fractional and integral frequency offset corrections. The fractional frequency offset $f_{F}$ is the offset in frequency that needs to be corrected to make all carriers lie on the center of the frequency bin. Assuming the transmitted signal is $s_{0}(t)$ and the received signal is $s(t)$, their relationship is given by

$$
s(t)=s_{0}(t) \exp \left\{-j 2 \pi f_{F} t\right\} \Rightarrow s(n)=s_{0}(n) \exp \left\{-j 2 \pi f_{F} n / f_{s}\right\}=s_{0}(n) \exp \{-j 2 \pi \varepsilon n / N\}
$$

where $\varepsilon=f_{F} / \Delta f$, which can be estimated by

$$
\tilde{\varepsilon}_{M L}=-\frac{1}{2 \pi} \arg \left\{\sum_{n=\tilde{\theta}_{M L}}^{\tilde{\theta}_{M L}+N_{C P}-1} s(n) s^{*}(n+N)\right\} \Rightarrow f_{F}=\tilde{\varepsilon}_{M L} \Delta f
$$

After obtaining the estimation of $f_{F}$, the signal can be de-rotated to compensate for the fractional frequency offset. However, when an integral frequency offset also exists, (5) should be changed to

$$
s(n)=s_{0}(n) \exp \left\{-j 2 \pi\left(f_{F}+f_{I}\right) n / f_{s}\right\}=s_{0}(n) \exp \{-j 2 \pi \varepsilon n / N-j 2 \pi m n / N\}
$$

where $m=f_{I} / \Delta f$ is an integer bigger than 0 . It can be learned that, since $0 \leq \tilde{\varepsilon}_{M L}<1, m$ cannot be estimated by (6). In such a case, Fourier transform may make each sub-carrier locate at incorrect frequency bin, introducing negative influences on the following processing as the prior knowledge of the carriers are all obtained according to the ISDB-T standard. To estimate the integral frequency offset $f$, the TMCC pilot carriers, which are always at the constant positions in different symbols, can be used to solve the following problem.

$$
m=\underset{m}{\arg \max } \sum_{i=0}^{N_{T M C C}-2} w(T[i]) s_{f}(T[i]+m) \cdot w(T[i+1]) s_{f}^{*}(T[i+1]+m)
$$

where $w$ is $4 / 3$ or $-4 / 3$ depending on $T[i], T[i]$ is the fixed position of the TMCC carrier, and $s_{f}$ is the frequency domain signal of the derotated received signal $s$ obtained by the Fourier transform. Based on the estimation of $m$, the frequency synchronization can be conducted. Then, 5617 active carriers and 4992 data carriers can be extracted from each OFDM symbol for the following processing.

\section{(3) Channel estimation and equalization}

Due to the influences of the propagation channel, the constellation of the ISDB-T signal has been polluted as 


$$
S(f)=H(f) \cdot S_{0}(f)
$$

where $S(f)$ is the extracted active carriers, $S_{0}(f)$ is the transmitted carriers, and $H(f)$ is the channel gain. To achieve the accurate modulated data, channel gain $H(f)$ estimation and equalization should be conducted. To do so, the SP carriers, which have predefined values, can be used. As the positions of SP carriers are changed from symbol to symbol, the symbol index should be firstly identified by

$$
p=\underset{p}{\arg \max } \sum_{s=0}^{N_{S p}-2} w(3 p+12 s) S_{f}(3 p+12 s) \cdot w(3 p+12 s+12) S_{f}^{*}(3 p+12 s+12)
$$

where $p=0,1,2$, or 3 is the symbol index, $w$ is $4 / 3$ or $-4 / 3$ depending on the position of SP carrier, and the value 12 is determined by the constant spacing of the SP carriers. With a known symbol index, the channel gain corresponding to SP carriers can be estimated by

$$
H_{S P}(f)=S(f) / S_{S P}(f)
$$

and the channel gain for all carriers can be estimated by the interpolation process. Then, the equalization process should be carried out to divide the estimated channel gain by the extracted active carriers. After channel equalization, the constellation of the transmitted signal can be more accurately estimated.

\section{(4) Symbol demapping}

Different from the communication application, where the transmitted bits should be recovered, the last step of reference signal demodulation for PBR applications is symbol demapping. In this paper, the hard decision method is used to estimate the value of each constellation point, while in noisy conditions the soft decision method is more preferable. In Sendai, two layers are used: layer A is QPSK modulated and layer B is 64QAM modulated. According to the ISDB-T standard, the carriers of layer A and layer B can be separated. For example, given one OFDM symbol of the sampled DTTB signal, the symbol demapping result is shown by Fig. 5, where layer $\mathrm{A}$ is indicated by the rectangles.

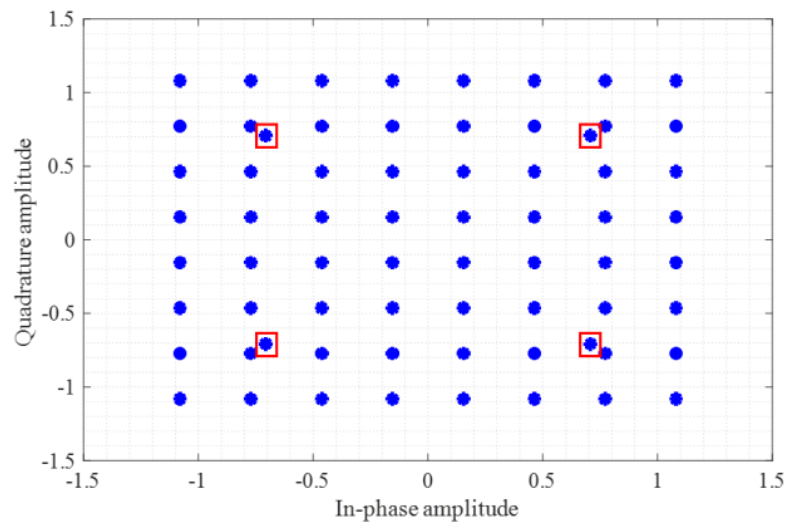

Figure 5 Demapping result of one OFDM symbol of the real-sampled DTTB signal.

\section{(5) Pilot signal remodulation and modification}

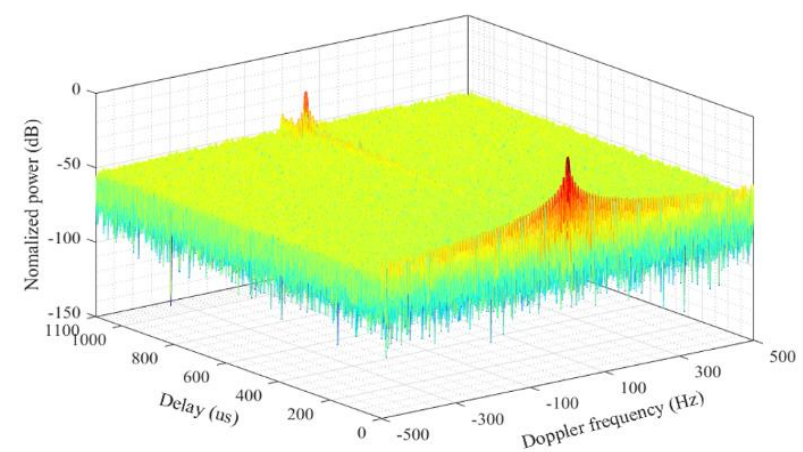

Figure 6 Cross-ambiguity diagram between the remodulated signal and the pilot-modified signal.

After reference signal demodulation, the influences of multipath signal and noise have been well mitigated, the remodulation can thus 
be conducted to get the ideal reference signal for PBR application. Moreover, a further step can be conducted by modifying the pilot carriers to suppress the undesired sidelobes in the range-Doppler domain. A simple and effective method is to reduce the power of the pilot carriers to $9 / 16(3 / 4 \times 3 / 4)$ [9]. In such a case, when cross-correlated with the surveillance signal, the power of the pilots will be changed to unity and the sidelobes can be canceled. For example, using the same dataset as used in Figure 3, the cross-ambiguity diagram between the remodulated reference signal and the pilot-modified reference signal is shown in Figure 6. It can be clearly seen that the sidelobes in the range and Doppler directions are suppressed and only the sidelobe at $1008 \mu$ s remains, which, however, is not considered here as the detected targets for many applications will not beyond $300 \mathrm{~km}$.

\section{(6) Surveillance signal processing}

As several processing steps have been carried out for the reference signal, the surveillance signal should also be processed to be time and frequency synchronized with the reference signal, i.e., OFDM acquisition and frequency offset correction should also be performed for the surveillance signal. Moreover, since the time and frequency synchronization cannot be perfectly conducted, a phase offset will remain. This phase offset can be modeled as linear with the frequency, i.e., $-2 \pi f \Delta \tau=-2 \pi k \Delta f \Delta \tau$, where $k=0,1, \ldots, N_{U}-1$. For the reference signal, because the channel equalization has been conducted, this phase offset has been canceled, as the phase offset is assimilated to the channel gain. For the surveillance channel, in order to detect the targets, the channel equalization cannot be conducted. Otherwise, the phase information of the moving targets will be damaged. Therefore, if directly cross-correlating the remodulated reference signal with the time/frequency synchronized surveillance signal without correcting the phase error, a time delay will be introduced. To estimate this phase offset, two simple methods can be used by conducting: (a) linear regression of the phases of the estimated channel gain; or (b) cross-correlation between the unequalized reference signal and the remodulated reference signal. Based on one of these two methods, the phase offset (time delay) can be determined, as shown in Figure 7, and then be used to correct the phase of the surveillance signal.
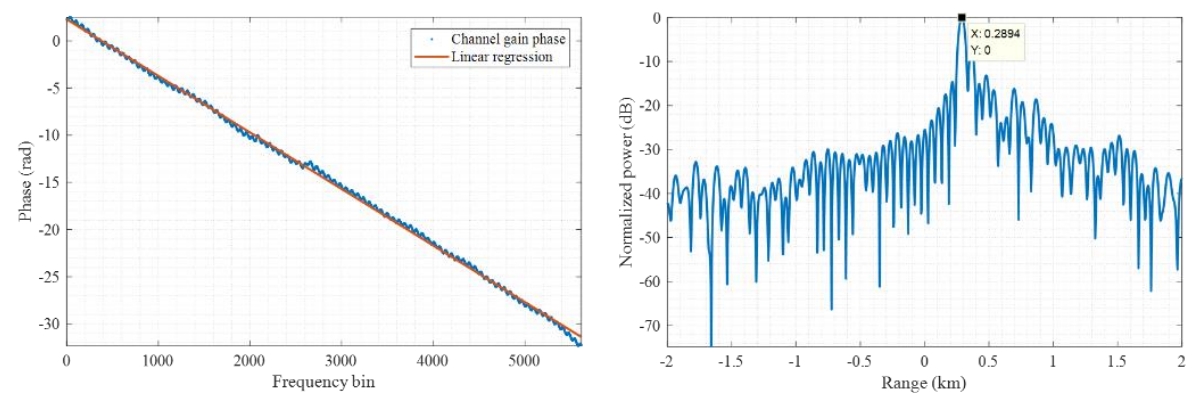

Figure 7. Phase offset estimation by (left) linear regression of the channel gain phase and (right) cross-correlating the unequalized reference signal with the reconstructed reference signal. The relationship between the regression coefficient $\alpha$ and the time delay $\Delta \tau$ is given by $\alpha=-2 \pi \Delta f \Delta \tau$.

\section{Experiment results and discussions}

To begin with, a simple experiment is conducted to validate the reference signal refinement, whose setup is shown in the left of Figure 8. Two Yagi-Uda antennas are faced to the TV tower, which is located at about $3 \mathrm{~km}$ from the experiment set. USRP B210 with a data sampling rate of $512 / 63 \approx 8.126 \mathrm{MHz}$ for both channels is used. One channel is acted as the reference and a multipath signal at about 5 $\mathrm{km}$ is added to the received signal. Another antenna is used to receive the surveillance signal. The 1D cross-correlation result is shown in the right of Figure 8, where the sidelobe at $5 \mathrm{~km}$ caused by the multipath signal and the sidelobes at $\pm 84 \mu s(25.2 \mathrm{~km})$ can be observed, which can be suppressed by the reference signal refinement process. By performing only reference signal reconstruction, the result is shown in the left of Figure 9. It can be seen that, although the multiple-path sidelobe has been suppressed, the sidelobes caused by the waveform properties remain. Besides, caused by the phase offset, the estimated delay does not correspond to the original result. By performing reference signal reconstruction, pilot modification, and phase offset correction at the same time, the result is shown in the right of Figure 9. It can be learned that sidelobes can be well suppressed and the delay between two channels can be accurately estimated. Moreover, an improved SNR can be obtained as the reference is pure, i.e., without noise, which is important for weak target detection. 

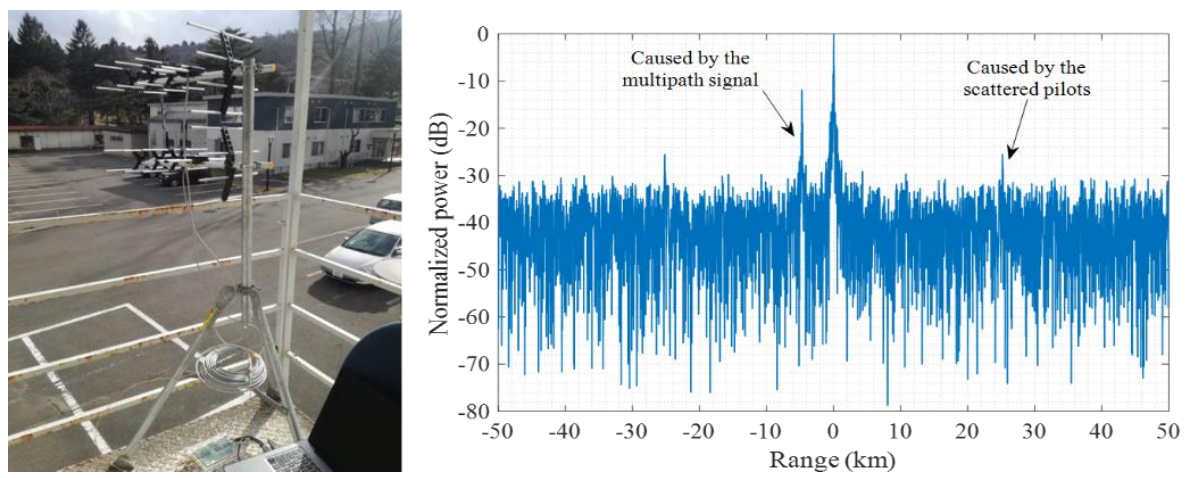

Figure 8. (Left) two Yagi-Uda antennas are both faced to the TV tower and (right) their cross-correlation result.
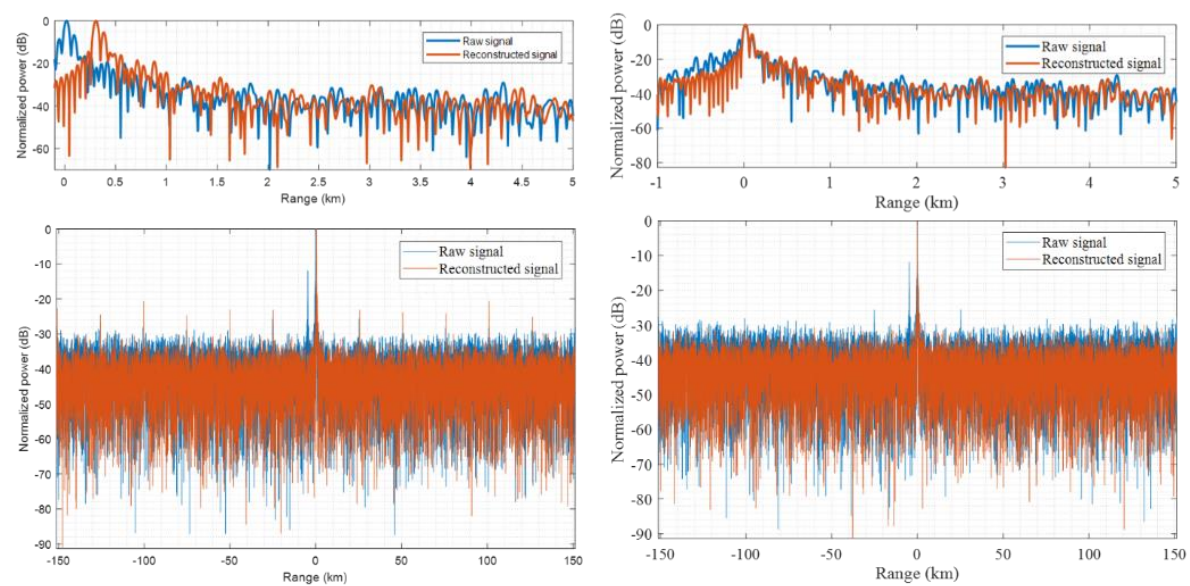

Figure 9. Cross-correlation results of (left) the remodulated reference signal and the surveillance signal and (right) the pilot-modified reference signal and the surveillance signal with phase correction. The top sub-figures are zoomed from the bottom sub-figures.
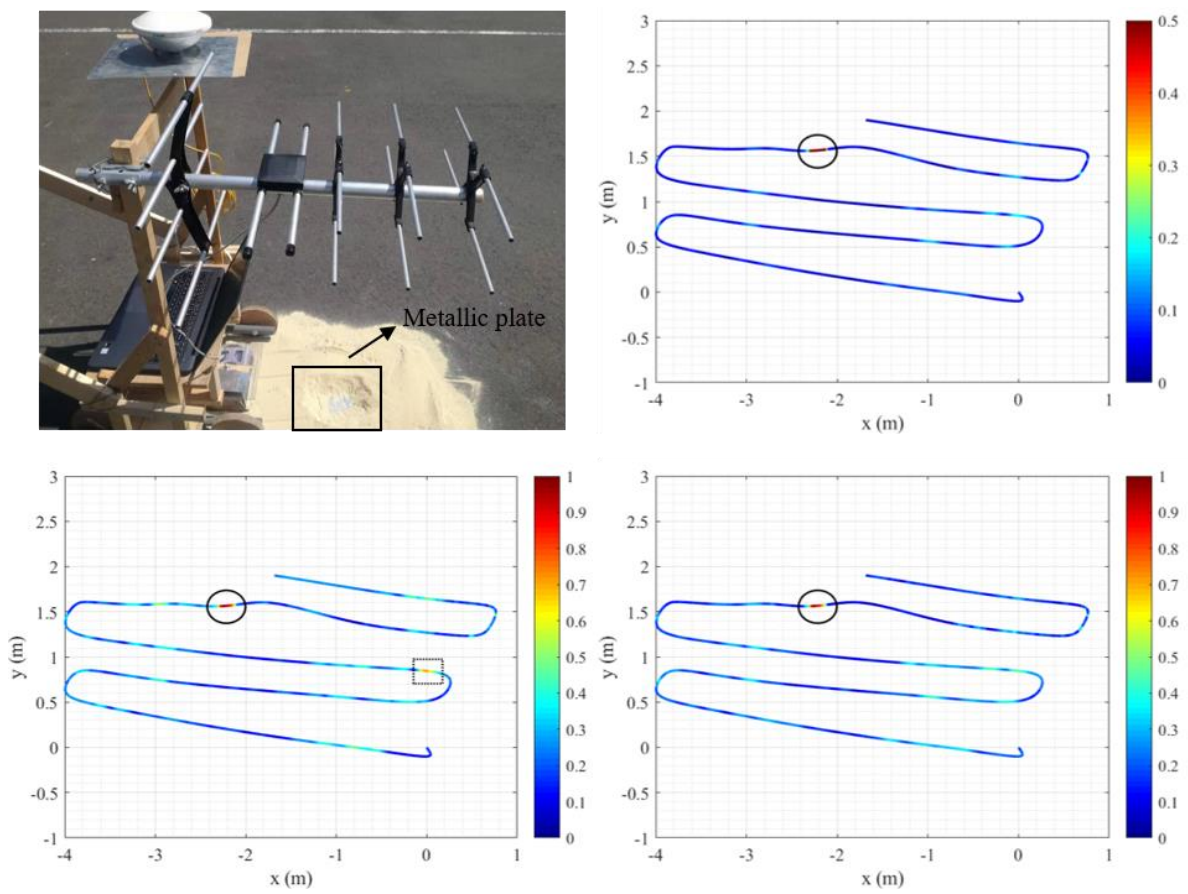

Figure 10. (Top left) the metallic plate buried in the sand is detected by the PBR system and its results obtained by (top right) surveillance signal auto-correlation, (bottom left) cross-correlation using raw signal, and (bottom right) cross-correlation with the refined signal. 
Then, with a total data acquisition time of about $82 \mathrm{~s}$ and a signal processing interval of about $0.09 \mathrm{~s}$, a metallic plate with the size of $10 \mathrm{~cm} \times 10 \mathrm{~cm}$ buried in the sand with a depth of $5 \mathrm{~cm}$ has been detected by the developed PBR system, as shown in the top left of Figure 10. The detection result obtained by the auto-correlation of the surveillance signal received by the Bowtie antenna is shown in the top right of Figure 10. It can be seen that the target position can be accurately determined, as indicated by the circle, because the received surveillance signal at the target position is stronger than those of other positions. However, auto-correlation cannot provide us range and phase information, which is important for the following processing stages. Therefore, the cross-correlation function between reference and surveillance signals is calculated. Without any refinement, the detection result is shown in the bottom left of Figure 10. Although the target can be detected, some artifacts will be generated, such as that indicated by the dashed rectangle. At last, by using the presented reference signal refinement method, the result is shown in the bottom right of Figure 10. It can be learned that, compared to its left subfigure, target position can be determined and fewer artifacts are generated.

In the current research step, only target detection has been conducted, while azimuth compression for 3D target imaging has not been implemented as the range resolution is low. In order to solve this problem, i.e., practically or virtually improve the range resolution, several potential solutions are provided here. Firstly, as demonstrated in [11], multiple DTTB channels can be combined to get a wider frequency bandwidth. In such a case, if the spectrum of different DTTB channels is continuous, then directly sampling all the channels for processing is sufficient to get a higher range resolution. If the spectrum is discontinuous, i.e., frequency gaps exist among different channels, some advanced methods, such as super spatially variant apodization (Super-SVA), compressive sensing (CS), and low-rank matrix completion (MC) based methods [11], should be applied to fill the frequency gaps in order to suppress the artifacts generated by the range compression. Secondly, since the targets may be located close to the ground surface, the target reflections are probably mixed with the ground reflection. In order to distinguish the targets from the clutter, a passive and wireless cooperative sensor designed by surface acoustic wave (SAW) delay lines can be used to attach to the target (such as gas or water pipe) [17]. The SAW sensor will convert an incoming electromagnetic signal into an acoustic signal whose velocity is much smaller and delay is thus much longer. Therefore, the target location can be accurately estimated without the interferences caused by the surroundings (e.g., the ground surface).

At last, in order to further improve the azimuth and range resolution, some super-resolution algorithms, such as CS algorithm and MUSIC algorithm, can be applied. For example, as the subsequent work of [7] which uses WiFi signal for subsurface target detection, a passive SAW sensor is buried in the sand and 2D imaging experiment is conducted, as shown in Figure 11. For this experiment, we note that: 1) The used WiFi transmitter is a commercial omnidirectional antenna, which is communicated with a WiFi access point (AP) to mimic the real WiFi environment; 2) the transmitted WiFi signal uses IEEE 802.11n standard with a central frequency of $2.422 \mathrm{GHz}$ and a bandwidth of $40 \mathrm{MHz}$, which is coupled to the oscilloscope to get the reference signal (i.e., no reference antenna is used); 3 ) the SAW sensor is connected to a commercial patch antenna, which has a $8 \mathrm{~dB}$ gain and a 60 degree radiation pattern; 4) the measurement antenna is a horn antenna and mounted on a rail to move above the buried sensor with a step of $2 \mathrm{~cm}$ and the synthetic aperture length is $50 \mathrm{~cm}$; 5) The height of the horn antenna is about $1 \mathrm{~cm}$ above the surface and the sensor is buried about $20 \mathrm{~cm}$ deep in the sand. The 2D imaging result obtained by the MUSIC algorithm is shown in the right of Figure 11. It can be seen that the SAW sensor has bistatic time delays from $1 \mu \mathrm{s}$ to $3 \mu \mathrm{s}$, which are well distinguished from the surface reflections. The angle of the sensor can be estimated by the MUSIC algorithm accurately with a high resolution.
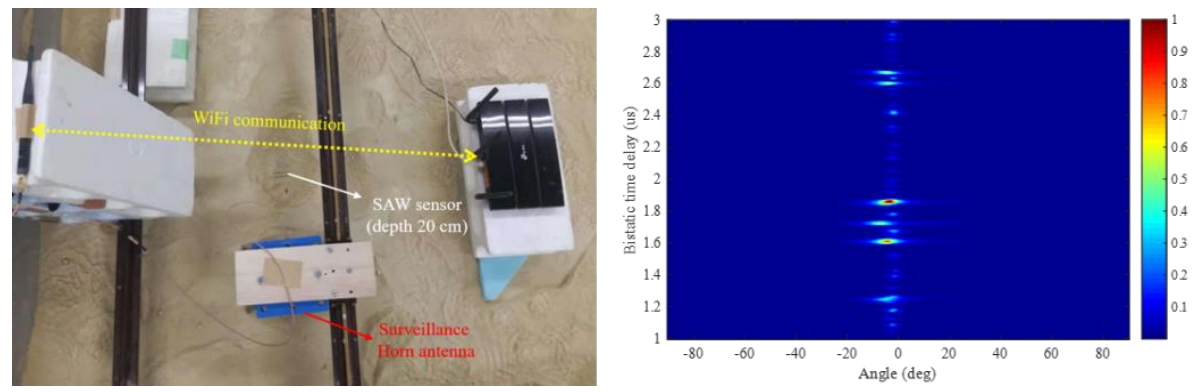

Figure 11. (Left) subsurface SAW sensor 2D imaging by using a WiFi-based PBR system, and (right) bistatic time delay and azimuth angle of the sensor can be effectively estimated by using the MUSIC algorithm. 


\section{Conclusions}

It has been validated in this paper that a PBR system with the DTTB signal can be used for subsurface target detection. The reference signal refinement has been proved to be effective to suppress noise, multipath echoes, and range-Doppler sidelobes. With respect to the limited resolution caused by the narrow bandwidth of the DTTB signal, some potential solutions have been proposed. In the next research step, more experiments will be conducted and advanced imaging methods will be studied in order to achieve the focused 3D image of the subsurface target.

\section{REFERENCES}

[1] P. E. Howland, H. D. Griffiths, and C. J. Baker, "Passive bistatic radar systems," in Bistatic Radar: Emerging Technology, New York, NY, USA: Wiley, 2008.

[2] H. D. Griffiths, and C. J. Baker, “An introduction to passive radar,” Norwood, MA, USA: Artech House, 2017.

[3] H. Kuschel, D. Cristallini, and K. E. Olsen, "Tutorial: Passive radar tutorial,” IEEE Aerosp. Electron. Syst. Mag., vol. 34, no. 2, pp. 2-19, Nov. 2019

[4] H. M. Jol, Ground penetrating radar theory and applications, Amsterdam, Netherlands: Elsevier, 2008.

[5] H. Liu, B. Xing, J. Zhu, B. Zhou, et al., "Quantitative stability analysis of ground penetrating radar systems," IEEE Geosci. Remote Sens. Lett., vol. 15, no. 4, pp. 522-526, 2018.

[6] R. Notarpietro, S. De Mattia, M. Campanella, et al., "Detection of buried objects using reflected GNSS signals," EURASIP Journal on Advances in Signal Processing, vol. 1, p.132, 2014.

[7] W. Feng, J.-M. Friedt, Z. Hu, et al., "WiFi-based imaging for GPR applications: Fundamental study and experimental results," in Proc. IET Int. Radar Conf., 2018, pp. 1-5.

[8] S. Searle, S. Howard, and J. Palmer, "Remodulation of DVB-T signals for use in passive bistatic radar," in Proc. 44th Asilomar Conf. Signals, Syst., Comput., Nov. 2010, pp. 1112-1116.

[9] H. Harms, L. Davis, and J. Palmer, "Understanding the signal structure in DVB-T signals for passive radar detection," in Proc. IEEE Radar Conf., 2010, pp. 532-537.

[10] W. Feng, J.-M. Friedt, G. Cherniak, et al., "Batch compressive sensing for passive radar range-Doppler map generation,” IEEE Trans. Aerosp. Electron. Syst., 2019, DOI: 10.1109/TAES.2019.2897474.

[11] W. Feng, J.-M. Friedt, G. Cherniak, et al., "Passive radar imaging by filling gaps between ISDB digital TV channels,” IEEE J. Sel. Top. Appl. Earth Obs. Remote Sens., vol. 12, no. 7, pp. 2055-2068, 2019.

[12] H. Liu, X. Xie, M. Sato, "Accurate thickness estimation of a backfill grouting layer behind shield tunnel lining by CMP measurement using GPR ," in 14th Int. Conf. on Ground Penetrating Radar, pp.137-142, 2012.

[13] T. W. Rondeau, "On the GNU Radio ecosystem," Opportunistic Spectrum Sharing and White Space Access: The Practical Reality, pp. 25-48, 2015.

[14] T. Takasu and A. Yasuda, "Development of the low-cost RTK-GPS receiver with an open source program package RTKLIB," in Int. Symp. GPS/GNSS, Jeju, Korea, Nov. 2009.

[15] F. Larroca, P. Flores-Guridi, G. Gomez-Sena, et al., “An Open and Free ISDB-T full seg Receiver Implemented in GNU Radio,” in WInnComm 16, pp. 1-10, 2016.

[16] J.-J. van de Beek, M. Sandell, and P. Borjesson, "ML estimation of time and frequency offset in OFDM systems," IEEE Trans. Signal Processing, vol. 45, no. 7, pp. 1800-1805, Jul 1997.

[17] W. Feng, J. -M. Friedt, G. Goavec-Merou, et al., "Passive radar delay and angle of arrival measurements of multiple acoustic delay lines used as passive sensors," IEEE Sensors Journal, vol. 19, no. 2, pp. 594-602, 2018. 